\title{
Subject Index Vol 61, 1993
}

Abortion 1

Accuracy 195

'ÄlS'S 105

Arteriovenous malformations 182

Biomechanics 49

Biopsy 207

Blood pressure 96

Brain imaging 32

lesions, nonenhancing 105

necrosis 118

neoplasms 32,182

tumor 39, 105 Cerebral palsy 49

Computed tomographic Scanner 39

- $\quad$ tomography 118

Computer 32

Cortex 102

Craniotomy 39, 80

Deep sensory neuron 12

Dopamine 1

Electrical Stimulation Parameters 129 Electrodes 60

Electroencephalography 32 Epidural space 60

- $\quad$ spinal cord Stimulation 60,129,

140, 146

Epilepsy 32

Floating line 207

Formalintest 171

Frameless stereotaxy 32

GABA 157

Glioma 105

Human fetal dopaminergic tissue 1

Hypothalamus 171

Imaging 195

Instrumentation 182

Laser surgery 182

Lemniscal sensory neuron 12 
Met-enkephalin 171

Microsurgery 80 •

Morphine 171

Motor disorders 60

Neural Stimulation 49

Neuroelectric implant 49

Neurotransmitter 157

Nucleus ventralis intermedius 12

Pain 102,129,140,146, 171

-, chronic 60

Paresthesiae 146

Parkinson's disease 1,118

Perception 129

Percutaneous thermocoagulation 96

Radiofrequency injury 118

Resection 39

Serotonin 157

Somatosensory evoked Potentials 49

System 12 Spinal cord 60

- Stimulation 60, 157 Stereophotogrammetry 207 Stereotactic biopsy 105

craniotomy 80,182

techniques 32, 80 Stereotaxis 24, 39 Stimulation Parameters 140, 146 Substance P 157

Surgical rehabilitation 49 Thalamotomy 24,118 Thalamus 12 , 118 Threshold 129 Trajectory angle 24 Transplantation 1 Tremor 12,24 Trigeminal neuralgia 96 Ultrasound 32 Usage ränge 140 